\title{
Análise de viabilidade de inserção de Fibra de Coco em Tijolos de Solo-Cimento
}

\author{
Feasibility analysis of insertion of Coconut Fiber in Soill-Cement \\ Análisis de viabilidad de inserción de Fibra de Coco en Ladrillos Suelo-Cimento
}

Recebido: 10/01/2022 | Revisado: 13/01/2022 | Aceito: 21/01/2022 | Publicado: 23/01/2022

\author{
Isamara Coelho Neves \\ ORCID: https://orcid.org/0000-0002-4425-5190 \\ Universidade Federal dos Vales do Jequitinhonha e Mucuri, Brasil \\ E-mail: isamara.coelho@ufvjm.edu.br \\ Karolayne Gonçalves Cota \\ ORCID: https://orcid.org/0000-0002-1987-5240 \\ Universidade Federal dos Vales do Jequitinhonha e Mucuri, Brasil \\ E-mail: ikarolayne.gonçalves@ufvjm.edu.br \\ Stênio Cavalier Cabral \\ ORCID: https://orcid.org/0000-0001-5241-9776 \\ Universidade Federal dos Vales do Jequitinhonha e Mucuri, Brasil \\ E-mail: istenio.cavalier@ufvjm.edu.br \\ Ismael Abu Kamel Marques de Oliveira \\ ORCID: https://orcid.org/0000-0002-1603-9036 \\ Universidade Federal dos Vales do Jequitinhonha e Mucuri, Brasil \\ E-mail: iismael.abu @ufvjm.edu.br
}

\begin{abstract}
Resumo
Com o passar dos anos a engenharia civil foi evoluindo em questão de técnicas construtivas e materiais práticos utilizados com o intuito de facilitar e baratear a obra. O impacto ambiental, quando começou a ser questionado, gerou muitas críticas ao setor de construção civil, pelo desperdício de materiais e dificuldade da destinação destes. O tijolo solo-cimento veio como uma alternativa sustentável para o meio ambiente, já que é considerado ecologicamente correto. Assim, este estudo tem como objetivo produzir tijolos de baixo custo, utilizando em sua composição fibras de coco e solo da região de Teófilo Otoni, a fim de discutir e avaliar a influência destes compostos nas propriedades do material e estudar sua viabilidade econômica e ambiental. Na metodologia deste trabalho foi realizada a confecção do tijolo solo-cimento com adição de fibras de coco. Foram confeccionados tijolos com e sem fibra com o intuito de realizar ensaios laboratoriais de compressão, a fim de discutir a influênciada fibra no tijolo. Com relação à resistência à compressão, a inserção das fibras possibilitou um ganho significativo, sendo que os tijolos com fibra apresentaram cerca de 4,5 vezes mais resistência que aqueles sem fibra. Portanto conclui-se que os tijolos fabricados suprem as expectativas desejadas, permitindo a produção de um material que se enquadre no padrão de preocupações com o meio ambiente, viabilidade de uma forma de emprego e o aumento da resistência à compressão.
\end{abstract}

Palavras-chave: Tijolo solo-cimento; Alternativa sustentável; Fibras de coco; Viabilidade.

\begin{abstract}
Over the years, civil engineering has evolved in terms of construction techniques and practical materials used in order to facilitate and cheapen the work. The environmental impact, when it began to be questioned, generated many criticisms of the civil construction sector, due to the waste of materials and difficulty in their disposal. The soil-cement brick came as a sustainable alternative for the environment, as it is considered ecologically correct. Thus, this study aims to produce low-cost bricks, using in its composition coconut fibers and soil from the Teófilo Otoni region, in order to discuss and evaluate the influence of these compounds on the material's properties and to study its economic and environmental viability. In the methodology of this work, the confection of the soil-cement brick with the addition of coconut fibers was carried out. Bricks with and without fiber were made in order to carry out laboratory tests of compression, in order to discuss the influence of fiber on the brick. Regarding compressive strength, the insertion of fibers allowed a significant gain, and bricks with fiber presented about 4.5 times more resistance than those without fiber. Therefore, it is concluded that the manufactured bricks meet the desired expectations, allowing the production of a material that fits the standard of concerns with the environment, feasibility of a form of use and increased compressive strength.
\end{abstract}

Keywords: Soil-cement brick; Sustainable alternative; Coconut fibers; Viability. 


\begin{abstract}
Resumen
A lo largo de los años, la ingeniería civil ha ido evolucionando en cuanto a técnicas constructivas y materiales prácticos utilizados para facilitar y abaratar la obra. El impacto ambiental, cuando comenzó a ser cuestionado, generó muchas críticas al sector de la construcción civil, por el desperdicio de materiales y la dificultad en su disposición. El ladrillo de suelo-cemento surgió como una alternativa sostenible para el medio ambiente, ya que seconsidera ecológicamente correcto. Así, este estudio tiene como objetivo producir ladrillos de bajo costo, utilizando en su composición fibras de coco y suelo de la región de Teófilo Otoni, con el fin de discutir y evaluar la influencia de estos compuestos en las propiedades del material y estudiar su viabilidad económica y ambiental. En la metodología de este trabajo se realizó la construcción del ladrillo suelo-cemento con la adición de fibras de coco. Se fabricaron ladrillos con y sin fibra para realizar ensayos de compresión en laboratorio, con el fin de discutir lainfluencia de la fibra en el ladrillo. En cuanto a la resistencia a la compresión, la inserción de fibras permitió una ganancia significativa, y los ladrillos con fibra presentaron alrededor de 4.5 veces más resistencia que aquellos sin fibra. Por tanto, se concluye que los ladrillos fabricados cumplen con las expectativas deseadas, permitiendo laproducción de un material que se ajuste al estándar de preocupaciones con el medio ambiente, factibilidad de unaforma de uso y mayor resistencia a la compresión.
\end{abstract}

Palabras clave: Ladrillo suelo-cemento; Alternativa sostenible; Fibras de coco; Viabilidad.

\title{
1. Introdução
}

A construção civil é uma importante atividade que traz benefícios, não apenas de caráter econômico, mas também de âmbito social, contribuindo para o contínuo desenvolvimento do país. Porém, em contrapartida, é um setor altamente degradante e os impactos ambientais causados podem ser observados em todas as etapas de sua cadeia produtiva (Laruccia, 2014).

Entretanto, como afirma Calvi (2018), hoje, com uma maior consciência da degradação ambiental ocorrida pelos processos que envolvem a construção, os usuários, órgãos públicos, construtoras e empresas estão atentos à preservação ambiental.

Segundo Cristina et al. (2018), a construção civil, através de uma educação ambiental, busca na atualidade estabelecer uma forma de progredir no estudo de novos materiais sustentáveis, atendendo as necessidades da sociedade com seu baixo custo e evitando a destruição ambiental.

Tendo em vista tal fato, entre as tecnologias empregadas, o tijolo de solo-cimento se mostra como uma alternativa muito útil (Filho, 2018). Esse tijolo é produzido a partir da prensagem de solo, cimento e água, destacando-se por apresentar uma menor agressão ao meio ambiente na sua fabricação, quando comparada aos tijolos convencionais (Lima, 2010).

De acordo com Cordeiro e Machado (2017) o tijolo de solo-cimento, conhecido como tijolo ecológico, se mostra como uma excelente opção construtiva, por possuir grandes vantagens ambientais. Além de utilizar de matéria-prima abundante e de baixo custo, ainda não precisa ser queimado, o que proporciona economia de energia em seu processo de fabricação e menor emissão de gás ao ambiente (Pisani, 2005).

O atual desafio da construção sustentável consiste em encontrar um tipo de resíduo, que ao ser incorporado ao composto de solo-cimento, ofereça melhoria das propriedades físico-mecânicas do material e ainda possibilite uma redução no consumo dos recursos naturais (Santos, 2016).

As fibras vegetais apresentam potencial para uso como elemento de reforço em compósitos, uma vez que sua adição reduz a fissuração na matriz, tendo em vista que as fissuras são interligadas e, como resultado, ocorre um aumento na tenacidade e na resistência à tração e ao impacto. Além disso, pelo fato do Brasil ser um país agroindustrial, a ideia da inserção de resíduos vegetais em matrizes frágeis parte da quantidade de resíduo disponível e da obtenção de produtos de baixo custo, alto desempenho e elevada durabilidade (Vilela, 2019).

O mercado de coco-verde tem crescido nos últimos anos no Brasil. Contudo, a casca de coco verde, subproduto do uso e da industrialização da água de coco, ainda é em grande parte depositada em lixões e aterros sanitários (Cardoso 
e Gonçalez, 2016). Os resíduos sólidos provenientes do consumo do coco in natura, cujas cascas são ricas em fibras formam amontoados em locais inadequados, degradando a paisagem, produzindo mau cheiro, e colocando em risco o meio ambiente e a saúde pública (Paz et. al., 2017).

Além disso, é importante citar que o coco caracteriza-se como um material de difícil decomposição, chegando a levarmais de 8 anos para se decompor. Deste modo, torna-se evidente a importância de se fazer a reutilização da casca do coco verde, pois além da importância das propriedades da sua fibra, existe a importância econômica e ambiental, visto que esses resíduos seriam descartados por não terem mais utilidade (Veloso et al., 2013).

Sendo assim, o presente trabalho tem por objetivo produzir tijolos de baixo custo e sustentáveis, utilizando em sua composição fibras de coco e solo da região de Teófilo Otoni - MG, a fim de discutir e avaliar a influência destes compostos nas propriedades do material e estudar sua viabilidade econômica e ambiental, proporcionando assim uma alternativa sustentável de produção de tijolos.

Visto que Teófilo Otoni está em uma das regiões com os mais baixos indicadores socioeconômicos de Minas Gerais, aelaboração de um tijolo de baixo custo e com produção totalmente artesanal é de grande valia para a população, pois permite que ela possa usufruir do solo da região e de materiais disponíveis gratuitamente para a fabricação do tijolo, além de contribuir para o meio ambiente dando uma destinação eficiente dos recursos oriundos da utilização do coco (Pereira e Souza, 2020).

\section{Tijolo Solo-Cimento}

Na produção dos tijolos, o solo é misturado ao cimento e à água em dosagens apropriadas, sendo esta mistura moldada em uma prensa, de onde obtém-se o tijolo prensado. Em sua técnica de fabricação o solo é o componente principal, com dosagem mínima de 10 unidades para uma de cimento (Nascimento et al., 2018).

Dentre os benefícios da utilização dos tijolos ecológicos está o fato deles poderem ser produzidos no próprio canteiro de obra, o que permite economia com transporte do produto, rapidez na fabricação, além de menor tempo de espera para a chegada ao local de utilização (Souza, 2006).

Porém, para que este produto possua qualidade comparável ao tradicional, de acordo com Pinto (2015), torna-se necessário um rigoroso estudo e processo de classificação do solo, visando a descrição de suas propriedades, a fim de garantir que ele possua características adequadas, que conduzam uma qualidade maior no tijolo. Além disso, é importante que durante o processo de produção seja dada atenção aos parâmetros de cura, densidade, tempo de compactação e proporções de solo e cimento corretos para que se atinja resistência requerida para o seu emprego.

Dentre as propriedades do solo, visando estabelecimento de critérios para o seu uso na construção, destacam-se a granulometria e índices de consistência e, em alguns casos a retração e compactação (Neves et al., 2009). No tocante ao tipode solo empregado Segantini e Wada (2011) afirmam que praticamente qualquer tipo pode ser utilizado, entretanto os solos mais apropriados são os que possuem teor de areia entre 45 e 50\%. Somente os solos que contêm matéria orgânica em sua composição não podem ser utilizados.

Com relação ao componente cimento, diversos tipos podem ser aplicados na mistura, desde que atendam as instruções normativas relacionadas. Porém, Souza et al., (2008) indicam que seja utilizado o CPII Z-32, por apresentar facilidade em ser obtido e possuir propriedades aceitáveis para emprego em tijolos de solo-cimento.

No que diz respeito à água, a ABNT NBR 10833:2012 (Fabricação de tijolo e bloco de solo-cimento com utilização de prensa manual ou hidráulica - Procedimento) indica que deve ser isenta de impurezas nocivas à hidratação do cimento. 


\section{Metodologia}

O presente estudo trata-se de uma pesquisa experimental e laboratorial, abrangendo ensaios de caracterização do solo utilizado, produção de tijolos de solo-cimento e ensaios laboratoriais para determinação de propriedades de resistência e absorção do material fabricado.

Para a fabricação do tijolo foi necessário efetuar retiradas de amostras de solo, que foram primeiramente submetidas a ensaios laboratoriais para classificação granulométrica. O terreno em que foram feitas as coletas está situado na região sudeste do Brasil, leste do estado de Minas Gerais, no município de Teófilo Otoni-MG, mais precisamente no Bairro Olga Correa, próximo à Universidade Presidente Antônio Carlos (UNIPAC), localizada na Rua Engenheiro Celso Murta, $\mathrm{n}^{\circ} 600$.

A fim de realizar a classificação granulométrica do solo, foi utilizado o Laboratório de Solos no Campus Mucuri da Universidade Federal dos Vales do Jequitinhonha e Mucuri, que dispõe dos equipamentos necessários, como peneiras, balanças de precisão, densímetros e agitador elétrico. A metodologia de ensaio foi desenvolvida com base nas normas ABNT NBR 6457:2016 (Amostra de solo - Preparação para ensaios de compactação e ensaios de caracterização) e a ABNT NBR 7181:2016 (Solo - Análise granulométrica).

Por outro lado, a preparação das fibras de coco e confecção dos tijolos ocorreu no Laboratório da Universidade presidente Antônio Carlos (UNIPAC), por este possuir prensa manual para produção dos tijolos, equipamento para realização dos testes de resistência à compressão do material fabricado e demais instrumentos requeridos para o processo.

Para que fosse possível utilizar a casca do coco como compósito foi necessário desfiá-la manualmente até que suas fibras estivessem totalmente separadas. Em seguida, estas foram deixadas submersas em água durante 5 dias, para posteriormente serem levadas à fervura por 5 minutos e por fim retiradas para secagem, a fim de eliminar o excesso de cera.

A confecção dos tijolos propriamente dita foi iniciada misturando solo previamente peneirado e cimento em um recipiente até que a mistura se apresentasse homogênea, com coloração uniforme, sem manchas de solo ou cimento, como mostra a Figura 1. Subsequentemente, foi adicionada água aos poucos e então foram inseridas as fibras do coco devidamente preparadas.

Observando os resultados da pesquisa desenvolvida por Filho (2018), foi adotado um traço de 1:0,1:0,6: 0,00507 (solo:cimento:água:fibra), em termos de proporções em massa.

Figura 1: Mistura manual do solo e cimento.



Fonte: Autores. 
Para o processo de fabricação do tijolo utilizou-se a prensa manual com dois furos. Conforme ilustra a Figura 2, a mistura foi inicialmente colocada no bocal da máquina (A), que direciona o material a um recipiente fechado (B), que quando movido, conduz até a forma (C) todo material de modo a preenchê-la completamente. Em seguida, após o fechamento do recipiente, puxa-se a alavanca da prensa e então ocorre a compressão do material e, por fim, a retirada do tijolo.

Figura 2: Prensa manual.



Fonte: Autores.

Os tijolos confeccionados apresentavam $25 \mathrm{~cm}$ de comprimento, $12 \mathrm{~cm}$ de largura, $8,5 \mathrm{~cm}$ de altura e dois furos com $6 \mathrm{~cm}$ de diâmetro, como mostra a Figura 3, conduzindo a um volume de $2070 \mathrm{~cm}^{3}$.

Figura 3: Tijolo de solo-cimento.

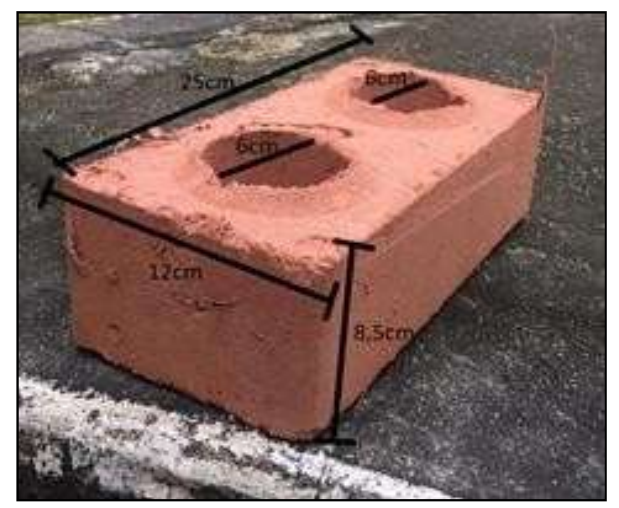

Fonte: Autores.

Com o intuito de avaliar e comparar os efeitos da inserção da fibra no material foram confeccionados tijolos com e sem a fibra de coco. Neste sentido, os produtos obtidos foram submetidos a ensaio de compressão simples e ensaio de absorção, em conformidade com a instrução normativa ABNT NBR 8492:2012 (Tijolo de solo cimento - Análise dimensional,determinação da resistência à compressão e da absorção de água - Método de ensaio).

\section{Resultados e Discussão}

Observa-se pelo Gráfico 1, que o solo utilizado é majoritariamente arenoso, com aproximadamente 77,94\% de areia $(0,06 \mathrm{~mm}-2,00 \mathrm{~mm}), 19,86 \%$ de silte $(0,002 \mathrm{~mm}-0,06 \mathrm{~mm})$, e $2,2 \%$ de pedregulho $(2,00 \mathrm{~mm}-60,00 \mathrm{~mm})$, conforme intervalos de diâmetro dos grãos dispostos na ABNT NBR 6502:1995 (Rochas e Solo - Terminologia). 
Gráfico 1: Curva de Distribuição Granulométrica do Solo.

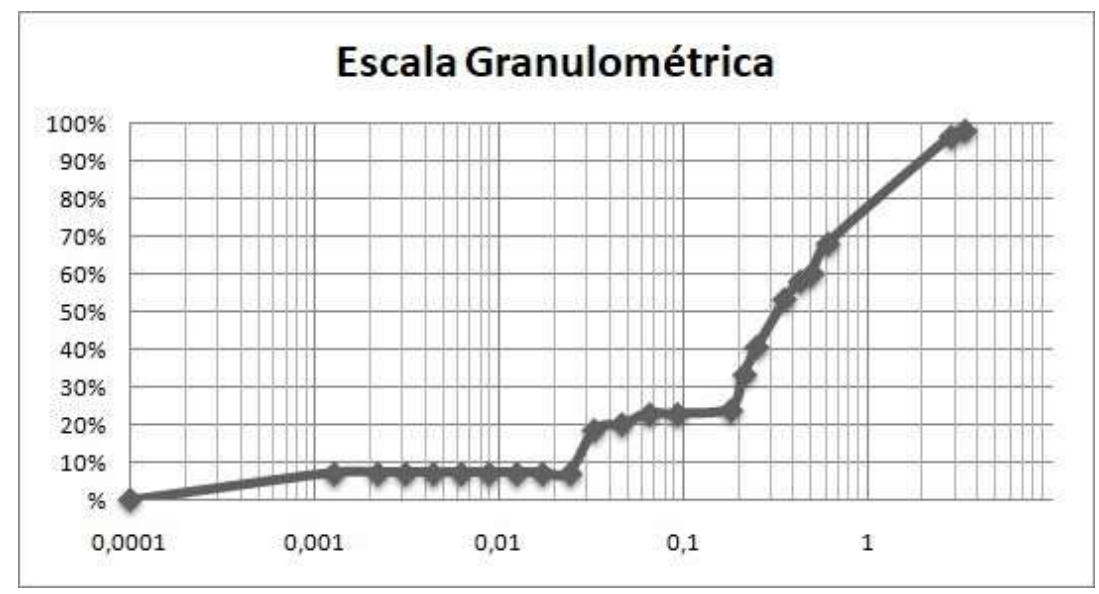

Fonte: Autores.

Com base nos dados obtidos, pode-se concluir que o solo local é arenoso, sendo composto principalmente de areia média. Ainda com base nestes dados percebe-se uma baixa porcentagem de silte e argila, demonstrando que o solo é majoritariamente formado por grãos grosseiros.

Com relação à resistência à compressão, a inserção das fibras possibilitou um ganho significativo, sendo que os tijolos com fibra apresentaram cerca de 4,5 vezes mais resistência que aqueles sem fibra. Ainda, é importante ressaltar que os valores alcançados atendem as exigências da ABNT NBR 8492:2012, onde é estabelecido que blocos vazados de solo-cimento semfunção estrutural devem apresentar resistência entre 1,7 MPa e $2 \mathrm{MPa}$. A Tabela 1 apresenta os valores obtidos no ensaio.

Tabela 1: Valores obtidos a partir do teste de compressão.

\begin{tabular}{ccc}
\hline Tijolo & Carga na ruptura $(\mathbf{k N})$ & Tensão na ruptura (MPa) \\
Sem fibra & 25,8 & 1,06 \\
Com fibra & 117,7 & 4,83 \\
\hline
\end{tabular}

Fonte: Autores.

Além disso, no que diz respeito à absorção, a Tabela 2 apresenta os valores individuais para os três testes realizados e a média correspondente.

Tabela 2: Valores de massa do tijolo seco e úmido.

\begin{tabular}{cccc}
\hline & Tijolo seco(g) & Tijolo úmido(g) & Absorção(\%) \\
I & 2786,3 & 3368,6 & 20,90 \\
II & 2780,5 & 3298,5 & 18,60 \\
III & 2784,3 & 3339,8 & 19,90 \\
\hline & & Média & 18,80 \\
\hline
\end{tabular}

Fonte: Autores.

Diante dos dados observados na tabela acima, conclui-se que os valores satisfazem os requisitos da NBR 10834:2012, que exige valores individuais inferiores a 22\% e a média destes valores máximos de $20 \%$. 


\section{Conclusão}

Com base nos resultados obtidos, percebe-se que a influência da adição de fibra foi positiva em relação à resistência acompressão, que é uma das principais propriedades que um tijolo deve possuir. Conforme observado, tanto os valores de resistência como de índice de absorção atendem à instrução normativa vigente e, portanto estão aptos para utilização.

Sendo assim, conclui-se que os tijolos fabricados suprem as expectativas desejadas, permitindo a produção de um material que se enquadre no padrão de preocupações com o meio ambiente, viabilidade de uma forma de emprego e o aumentoda resistência à compressão.

Assim, tem-se que a fabricação do tijolo solo-cimento com adição de fibra é de grande valia tanto para o Município deTeófilo Otoni e quantas demais cidades, sendo útil para a preservação do meio ambiente com a redução do lixo gerado pelo consumo do coco, além de gerar uma fonte de renda caso seja utilizado para produção e venda, ou ainda, reduzindo custos paraquem pretende fabricar o próprio tijolo de sua construção.

Com sugestão para pesquisas futuras, propõe-se a realização de análise comparativa entre os tijolos de solocimento comuns e aqueles com inserção de fibra, no que diz respeito à redução de fissuras na matriz.

\section{Referências}

ABNT. (1995). NBR 6502: Rochas e Solos - Terminologia.

ABNT. (2016). NBR 6457: Amostra de solo- Preparação para ensaios de compactação e ensaio de caracterização.

ABNT. (2016). NBR 7181: Solo - Análise Granulométrica.

ABNT. (2012). NBR 8492: Tijolo de Solo Cimento - Análise Dimensional, determinação da Resistência à Compressão e da Absorção de Água - Método de Ensaio.

ABNT. (1989). NBR 10833: Fabricação de tijolo maciço e bloco vazado de solo-cimento com a utilização de prensa hidráulica. Procedimento.

Calvi, L. F. H. (2018). Sustentabilidade na Construção Civil: Estudo de caso em uma organização não governamental. Monografia de Graduação, Universidade Federal do Rio de Janeiro, Rio de Janeiro, Brasil. http://repositorio.poli.ufrj.br/monografias/monopoli10023720.pdf.

Cardoso, M. S., \& Gonçalez, J. C. (2016). Aproveitamento da casca do coco-verde (Cocos Cucifera L.) Para produção de polpa celulósica. Revista Ciência Florestal, 26(1), 321-330. https://www.scielo.br/j/cflo/a/D3ZKqf8yW3YTpLyLbHdr9FP/?format=pdf\&lang=pt.

Cristina, P., Salomão, P. E. A., Cangussú, L., \& Carvalho, P. H. V. de (2018). Tijolo solo cimento com adição de fibra vegetal: uma alternativa na construção civil. Revista Research, Society and Development, 7 (9), 01-18.

Cordeiro, C. R., \& Machado, V. C. (2017). Sustentabilidade na fabricação do tijolo solo-cimento: estudo comparativo com blocos convencionais. Monografia de Graduação, Instituto Federal de Educação, Ciência e Tecnologia de Goiás, Aparecida de Goiânia, Goias, Brasil. https://repositorio.ifg.edu.br/bitstream/prefix/140/3/tcc_Cristine\%20Cordeiro_Vanessa\%20Machado.pdf.

Filho, F. E. D. (2018). Utilização da fibra de coco como proposta de substituição de cimento em tijolos ecológicos de solo-cimento: uma análise físicomecânica. Monografia de Graduação, Universidade Federal Rural do Semi-árido, Mossoró, Rio Grande do Norte, Brasil. https://repositorio.ufersa.edu.br/bitstream/prefix/4931/1/FranciscoEDF_MONO.pdf

Laruccia, M. M. (2014). Sustentabilidade e impactos ambientais da construção civil. Revista ENIAC Pesquisa, 3 (1), 69-84. https://ojs.eniac.com.br/index.php/EniacPesquisa/article/view/124.

Lima, R. C. O. (2010). Estudo da durabilidade de paredes monolíticas e tijolos de solo-cimento incorporados com resíduo de granito. Dissertação de mestrado, Universidade Federal de Campina Grande, Paraíba, Brasil. https://bdtd.ibict.br/vufind/Record/UFCG_f7479e2d2fa878d8be79185877ebde2e.

Nascimento, Á. M., Feitosa, A. O., Almeida, T. S., \& Lacerda, D. M. (2018). Tijolo modular de solo-cimento como material na construção civil. Revista INTERSCIENTIA, 6 (1), 187-202.

Neves, C. M. M., Faria, O. B., Rotondaro, R., Cevallos, P. S., \& Hoffmann, M.V. (2009) Seleção de solos e métodos de controle na construção com terra práticas de campo. 2009. Rede Ibero-americana PROTERRA. http://redeterrabrasil.net.br/wp-content/uploads/2020/08/Sele\%C3\%A7\%C3\%A3o-de- solos-em\%C3\%A9todos-de-controle-na-constru\%C3\%A7\%C3\%A3o-com-terra.pdf.

Paz, E. C. S. da, Pedroza, M. M., Oliveira, L. R. A. de, \& Paz, R. R. S. de. (2017). Alternativa de Exploração Sustentável dos Resíduos do Coco Verde para a produção de energia. Revista Brasileira de Energias Renováveis, 6 (2), 318-345.

Pereira, N. J., \& Souza, K. R. de. (2020). Pobreza no estado de Minas Gerais: Uma análise da região Norte. Revista Iniciativa Econômica, 4 (2). 
Research, Society and Development, v. 11, n. 2, e22311225549, 2022

(CC BY 4.0) | ISSN 2525-3409 | DOI: http://dx.doi.org/10.33448/rsd-v11i2.25549

Pinto, L. M. (2015). Estudo de tijolos de solo cimento com adição de resíduo de construção civil. Monografia (Graduação) - Curso de Engenharia Civil, Universidade Federal de Santa Maria, Santa Maria, Brasil.

Pisani, M. A. J. (2005) Um material de construção de baixo impacto ambiental: o tijolo de solo-cimento. Revista SINERGIA, 6 (1), 53- 59.

Santos, A. M. (2016). Avaliação de propriedades físico-mecânicas do composto solo-cimento com incorporação de fibra de ráfia para produção de tijolos ecológicos. Monografia de Graduação, Universidade Federal do Recôncavo da Bahia, Cruz das Almas, Bahia, Brasil. https://www2.ufrb.edu.br/bcet/components/com_chronoforms5/chronoforms/uploads/tcc/20190314171943_2015.1_-_TCC_dila_Michele_Santos_-

_Avaliao_De_Propriedades_Fsico-Mecnicas_Do_Composto_Solo-Cimento_Com_Incorporao_De_Fibra_De_Rfia_Para_Produo_De_Tijolos_Ecolgicos.pdf.

Segantini, A. A. S. M. da, \& Wada, P. H. (2011). Estudo de dosagem de tijolos de solo-cimento com adição de resíduos de construção e demolição. Revista Acta Scientiarum. Technology, 33 (2), 179-183.

Souza, M. I. B. de. (2006). Análise da adição de resíduos de concreto em tijolos prensados de solo-cimento. Dissertação (Mestrado) - Curso de Engenharia Civil, Universidade Estadual Paulista, Ilha Solteira, São Paulo, Brasil.

Souza, M. I. B. de, Segantini, A. A. S., \& Pereira, J. A. (2008). Tijolos prensados de solo-cimento confeccionados com resíduos de concreto. Revista Brasileira de Engenharia Agrícola e Ambiental. 12 (2), 2015-212.

Veloso, Y. M. S. da, Souza, I. E. S., Santos, J. V. dos, \& Leite, M. S. (2013). Reutilização da fibra da casca do coco verde para a produção de materia prima industrial. Revista O caderno de graduação das ciências exatas e tecnológicas - CGCET, 1 (17), 91-98.

Vilela, A. P. (2019). Tijolo solo-cimento reforçado com partículas vegetais e resíduo de mineração. Tese de Doutorado, Universidade Federal de Lavras, Lavras, Minas Gerais, Brasil. 